\title{
Product-Customization and Payment Policy with Postponement Strategy
}

\section{Jian-Qiang Luo}

School of Management, Jiangsu University, Zhenjiang, China.

Email: ljq2809@163.com

Received October $24^{\text {th }}, 2012$; revised November $27^{\text {th }}, 2012$; accepted December $10^{\text {th }}, 2012$

\begin{abstract}
Manufacturers adopting postponement strategy can lower operational cost and quickly respond customers' personalized demands. Adopting postponement strategy, however, has often led manufacturers to situations of higher risk of holding exclusive material in the customization stage. For example, customers prefer to order flexibly from manufacturers so as to be able to respond flexibly to their custom changes. Manufacturers, on the other hand, prefer customers to place full orders as early as possible so that they can hedge against the risks of under-production, especially in the customer order decoupling point (CODP) downstream. Motivated by this problem, we model a dynamic cost model in this paper to evaluate the risks and benefits. Using the Logistic curve, we develop a payment policy for manufacturer adopting postponement strategy in the CODP downstream. Our research demonstrates that, compared with the payment policy of product completed, payment in stages according to product-customization can coordinate conflict between manufacturers and customers in the process of adopting postponement strategy manufacturing system. We also discuss variation of CODP positioning, total cost and payment when rate of return changes.
\end{abstract}

Keywords: Postponement Strategy; Customer Order Decoupling Point (CODP); Payment Policy; Dynamic Cost; Logistic Curve

\section{Introduction}

Over the past decade, most manufacturing industries have witnessed an unprecedented variety of products, while product lifetimes have continued to decrease [1]. The move towards low cost, high service and quick response to meet customers' personalized demands has become a notable trend. However, due to global economic integration, the competitiveness of companies becomes fiercer and the customers demand uncertainty become more increasing, manufacturing enterprises have been facing many new challenges. To survive and be successful despite market turbulence, many manufacturing enterprises have adopted postponement strategy, such as Xilinx, Hewlett-Packard, Dell, Mars, Motorola, Gillette, Benetton [2-4]. Such a strategy has been widely accepted to be an effective way to reduce the operation risks associated with product variety by exploiting the commonality between items and by designing the production and distribution processes to delay the point of differentiation which is also called customer order decoupling point (CODP) [5]. Adopting postponement strategy can increase flexibility, reduce uncertainties and decrease the costs of complexity by reducing the variety of components and processes within the system [6,7].
However, postponement is not an omnipotent strategy. The main drawback of postponement strategy is the higher cost and risk of holding customization components/product [8]. Thus, decision of product-customization and payment policy with postponement strategy between manufacturers and customers is an important issue, especially in China. Customers prefer to order flexibly from manufacturers so as to be able to respond flexibly to their custom changes. Manufacturers, on the other hand, prefer customers to place full orders as early as possible so that they can hedge against the risks of under-production and compensate fund shortage, especially for the small and medium-sized enterprise. Motivated by this situation, our paper is primarily concerned with several research questions as follows. The first question is how model the dynamic cost considering the risks of material resource commonality diminishing in postponement strategy. The second question is where position the CODP under the coexistence of customization orders based on time value of money. The third question is how determine payment policy for customers on the based of the optimal CODP positioning.

The remainder of this paper is organized as follows. Section 2 reviews related literature and discusses the 
motivation of this research. Section 3 presents the theory hypothesis of product-customization and payment policy with postponement strategy. Section 4 gives some notations and basic assumptions of the research topic. Then, in Section 5, we model both dynamic cost and payment policy as logistic curve equation. Furthermore, efficient algorithms to determine payment policy are developed. The empirical analysis and sensitivity analysis are carried out in Section 6. Section 7 concludes the paper.

\section{Literature Review}

To respond quickly to changes in the market and to insure that the response will be able to satisfy customer fashion requirements, postponement strategy is one of the most important methods [9]. The concept of postponement strategy has a long history, not only in the academic literature, but also of practical application [10]. Postponement strategy is considered as an organizational concept, whereby some of the activities in the supply chain are not performed until customer orders are received [3]. During that evolution of the issue, it has been well documented where, when, and how to implement postponement strategy $[4,11]$. For a more detailed review, we also refer the readers to Van Hoek (2001) and Biao Yang (2004).

Faced with constant changes (e.g. volume change, variety change and delivery change), it is a natural option for a manufacturers to seek opportunities for delaying some activities like the final processing or manufacturing of product to as late a time as possible [4]. Postponement can enhance a company's flexibility to effectively meet the requirements of the growing product variety and quick response [12]. With the development of technology, especially information technology and manufacturing technology, postponement is increasingly appealing and available in manufacturing area. The concept of postponement, as applied to manufacturing, could be interpreted as retaining the product in a neutral and noncommitted status as long as possible in the manufacturing process. Using manufacturing postponement a manufacturer could perform the non-postponed activities speculatively in a make-to-stock (MTS) fashion and use reactive capacity only to customize the final product thereby enhancing the profitability of accurate response [13]. This is well-known that make-to-stock (MTS) manufacturers can fill customer orders quickly, but face inventory risks associated with short product life cycles and variability in demand, while make-to-order (MTO) manufacturers can provide product variety and custom orders with lower inventory risks, but generally have longer customer lead times [14]. Customer order decoupling point (CODP) employs a hybrid approach in which a product is partially manufactured via MTS and then completed via MTO only after a customer order is placed, which can increase the flexibility a manufacturer has to deal with uncertainties in market demand. It was discussed by Lee (1996) and Lee and Tang (1997) [15,16].

To clearly position the essential contributions of this paper, we essentially address the issues of product-customization cost and customer payment. There is a large body of literature on cost optimization of implementing postponement strategy, e.g. Chung Yeh, Hung-Cheng Yang (2003) [17] constructed original and postponed garment dyeing cost models and use practical parameter data to simulate various situations, and found that the cost of the postponement model is lower than the cost of the original model when key parameters are large. Adopting postponement strategy according to customers' specifications may be an important competitive advantage, but Haapasalo (2004) [18] pointed out that while product-customization enables manufacturers to charge premium prices, it also exposes them to new kinds of uncertainties. A particularly difficult challenge is coping with customers who request changes in specifications during the fulfillment of their orders. Recently, Jukka and Antti (2012) [19], proposed it exposed manufacturers to the risk of customers requesting changes in their specifications during the fulfillment of their orders, which manufacturers incur additional costs. So manufacturers may tend to apply postponement upstream, because raw material inventories are usually cheaper than the end item inventories.

Due to the paucity of research on between product-customization and payment policy with postponement strategy, our paper contributes to the extant literature in several ways. We provide the dynamic cost model which considers material holding risk and customer payment model under postponement strategy, simultaneously. In addition, we show how product-customization affects customer payment. The first is the dynamic cost model, which includes the cost of CODP upstream and CODP downstream. The second is the customer payment model. It is important significance for integrate two models to solve three problems in Section 1. The models detail and results are presented in Section 4.

\section{Problem Description}

The objective of manufacturer adopting postponement strategy is to quickly respond customers' customization demands with low cost, and enhance the competition ability of manufacturing services. However, after manufacturers receive the customer orders and acquire demand information, the exclusive of material resource would become increasing, as CODP downstream products customization process continue. Meanwhile, the manufacturers assume the greater material holding risk because of 
customer demand information ambiguity. Manufacturers have to adopt the service mode of payment in stages in the CODP downstream, namely product-customization, according to the orders fulfillment conditions, which can ease customers' concerns, such as product design, production and service quality. Manufacturers can win more valuable orders ultimately. At CODP (customer place order), the manufacturer charge the first deposit, as a continuation of the product customization, charge payments in stages for customers, when products are delivered, surplus is cleared. Such as furniture manufacturers have adopted this model, specifically as shown in Figure 1. Theoretical value of such problems has two aspects:

- Manufacturers could effectively prevent from order changes due to customers demand uncertainty. The risk of material resources waste can be effectively guaranteed by the manufacturers in the CODP downstream.

- According to customization effect, customers could ensure the customization demands to achieve by payment in stages.

As shown in Figure 1, manufacturers produce common components or modules in the CODP upstream, which is characterized by high degree of neutral, components with a wide range of usage and low holding risk; while manufacturers customize product according to customer order specific content in the CODP downstream. Along with process of product-customization, the exclusive of material resource to meet customer requirements is more and more strong, material hold risk is more and more high. At this time, manufacturers need adopt payment in stages to compensate for or offset enterprise material holding risk in the process of customer real-time involvement. If we consider production costs of common component and customization production, respectively, coming from CODP upstream and downstream, common components production costs have been paid in advance by manufactures in the CODP, enterprise profit is negative, but with very low materials holding risk. Customers will pay in stages to compensate for manufactures' holding risk only if customization contents are confirmed. Manufacturers maybe gradually gain.

Many manufacturing enterprises to implement postponement strategy are based on the following assumption: The investment to common or module components in the CODP upstream must be compensated after products are customized in the CODP downstream. But manufacturers hold higher risks due to material exclusiveness enhancement and the replacement cost increasing in the customization production process, so manufacturers should regard the customization production (customer information materialized) process as a discount rate borrowing as scheduled. When each customization process customers participating is completed, the customer should pay the corresponding price in accordance with service rules established. In that way, the change rule of benefits subtracted costs is from negative to positive.

\section{Notations and Assumptions}

We will begin the modeling process by presenting the following notations and assumptions.

\subsection{Notations}

The notations employed in the paper are as follows:

$i$ : product type number, $i \in[1, N]$, adopting postponement strategy manufacturing system can provide $N$ products;

$r$ : rate of return (ROI), which both customers and manufacturers are able to accept;

$t$ : time point when product is in the implementing postponement strategy manufacturing system, $t \in\left[0, L T_{i}\right]$;

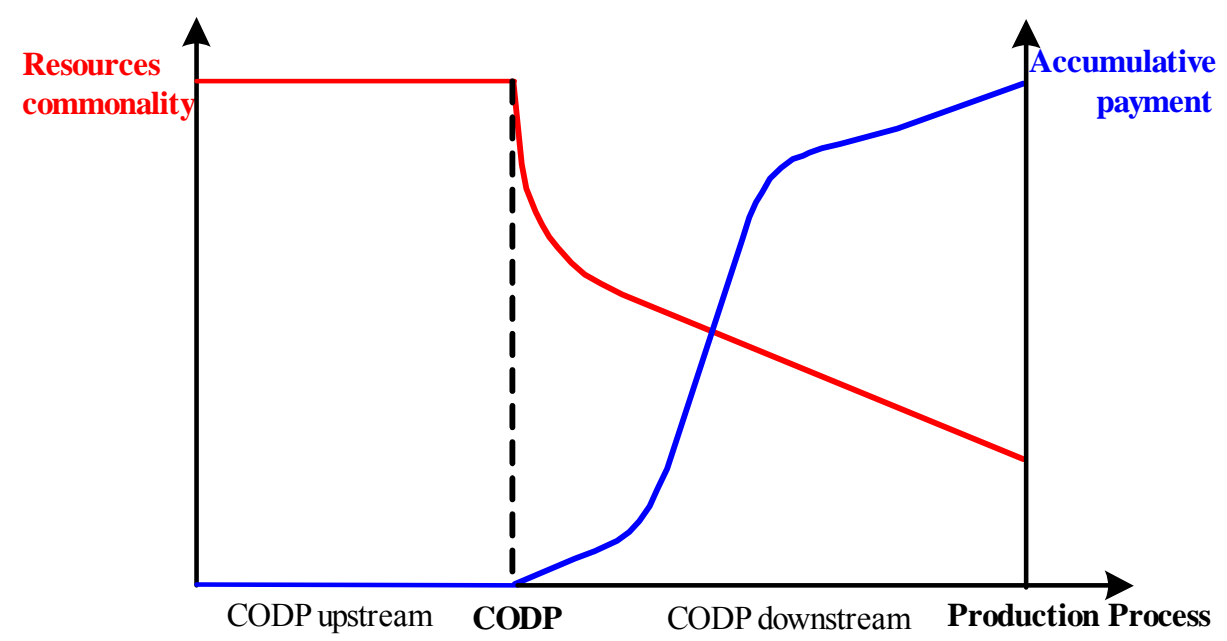

Figure 1. The relationship of resource commonality and accumulative payment in postponement strategy. 
$L T_{i}$ : lead time of $i$ order. Material resource is in the CODP upstream when $t \in[0, \mathrm{CODP}]$, otherwise, is in the CODP downstream when $t \in\left(\mathrm{CODP}, L T_{i}\right]$;

$D$ : total quantity of common components in the CODP upstream;

$p_{0}$ : unit price of the common components of raw materials;

$c_{1, t}$ : unit man-hour costs of common components in the CODP upstream at $t$;

$p_{i}$ : unit price of the $i$ finished product;

$c_{2, i, t}$ : unit man-hour costs of the $i$ custom product in the CODP downstream at $t$;

$d_{i}$ : customer demand quantity of the $i$ product;

$p_{i, j, t}$ : Accumulative payment of customer $j^{\text {th }}$ payment for the $i$ product at $t$;

$P_{i}$ : the $i$ custom order sales price, $P_{i}=p_{i} d_{i}$

$a_{i}$ and $b_{i}$ : unknown parameters of the $i$ custom order respectively.

\subsection{Assumptions}

The assumptions employed in this paper are stated as follows.

1) CODP positioning is relative to lead time, we believe the lead time including $[0, \mathrm{CODP}]$ and (CODP, $L T]$, which indicate the process time in the CODP upstream and downstream, respectively;

2) A type of common component in the CODP upstream is provided for customization differentiated in the CODP downstream;

3) Raw material purchase quantity of common component is exactly equal to $N$ customization production line demands, and the correlation of demand quantity between common component and finished product is 1:1, namely, $D=\sum_{i=1}^{N} d_{i}$;

4) Each process is equilibrium, and the processing time and labor-hour cost of each process is same;

5) Customer must pay initial payment when customer place his order, the initial payment is equal to advanced cost in the CODP upstream by manufacturer;

6) At some point, the second payment price is equal to the total cost of implementing postponement strategy manufacturing system.

\section{Model Formulation}

Applying notations and assumptions defined previously, the dynamic cost model and customer payment model for this problem is described as follows.

\subsection{Dynamic Cost Model}

According to the postponement strategy implementation mechanism, common components which replacement cost is low and neutral degree is high are produced by manufacturer in the CODP upstream. Costs mainly include procurement costs and production cost of common components. So CODP upstream static cost is:

$$
T C_{0 \rightarrow \mathrm{CODP}}=\sum_{t=0}^{\mathrm{CODP}}\left(p_{0}+c_{1, t}\right) D \text {. }
$$

According to the discrete customers individual demands, products which replacement cost is high are customized by manufacturer in the CODP downstream. Due to high risk of customization production, the customers need pay in stages to compensate for the material holding risk. So CODP downstream static cost is:

$$
T C_{\mathrm{CODP} \rightarrow L T}=\sum_{i=1}^{N} \sum_{t=\mathrm{CODP}}^{L T_{i}} c_{2, i t} d_{i} .
$$

If the risks of manufacturers operation and the dynamics of implementing postponement strategy are both considered, future customization problem should be integrated into the current decision-making at CODP. Formula (1) and (2) is converted to future value and present value at CODP respectively, this will help enterprises make decision of CODP positioning, then the present value of customized product total cost at CODP is:

$$
\begin{aligned}
T C= & \sum_{t=0}^{\text {CODP }}\left(p_{0}+c_{1, t}\right) D(F / P, r, t) \\
& +\sum_{i=1}^{N} \sum_{t=\mathrm{CODP}}^{L T_{i}} c_{2, i t} d_{i}(P / F, r, t) .
\end{aligned}
$$

The first term of formula (3) occurs in the CODP upstream. Even if the holding risk of common components is low, but manufacturers backlog a certain amount of circulating fund and come up "materialized labor", so it has certain significance for cost to be converted to future value at CODP. The second term of formula (3) occurs in the CODP downstream. The costomization processes, which have dynamic, require customers to pay in stages for compensattion, because the exclusivity of common components increases with the customers' order completion. The cusomization cost in the CODP downstream is converted to CODP, which contributes the manufacturing enterprise withdrawing funds.

Time has continuity, so the formula (3) can be rewritten as formula (4):

$$
\begin{aligned}
T C= & \int_{0}^{\operatorname{CODP}}\left(p_{0}+c_{1, t}\right) D(1+r)^{t} \mathrm{~d} t \\
& +\sum_{i=1}^{N} \int_{\operatorname{CODP}}^{L T_{i}} c_{2, t t} d_{i}(1+r)^{-t} \mathrm{~d} t .
\end{aligned}
$$

Assumption of implementing postponement strategy manufacturing system exists as in steady state [20], namely, $c_{1, t}$ and $c_{2, i t}$ are constants which are expressed as $c_{1}$ and $c_{2, i}$ as follows, then the formula (4) 
can be expressed as:

$$
\begin{aligned}
T C= & \int_{0}^{\operatorname{CODP}}\left(p_{0}+c_{1}\right) D(1+r)^{t} \mathrm{~d} t \\
& +\sum_{i=1}^{N} \int_{\mathrm{CODP}}^{L T_{i}} c_{2, i} d_{i}(1+r)^{-t} \mathrm{~d} t .
\end{aligned}
$$

Let

$$
\begin{aligned}
\left.T C^{\prime}\right|_{\mathrm{CODP}}= & \left(p_{0}+c_{1}\right) D(1+r)^{\mathrm{CODP}} \\
& -\sum_{i=1}^{N} c_{2, i} d_{i}(1+r)^{-\mathrm{CODP}}=0,
\end{aligned}
$$

we can get:

$$
\mathrm{CODP}^{*}=\frac{\ln \sum_{i=1}^{N} c_{2, i} d_{i}-\ln D\left(p_{0}+c_{1}\right)}{2 \ln (1+r)} .
$$

It is obviously that $\left.T C^{\prime \prime}\right|_{\text {CODP }}>0$, namely, when CODP $=$ CODP $^{*}$, the dynamic total cost of implementing postponement strategy manufacturing system has minimum value.

\subsection{Customer Payment Model}

In the CODP downstream, customization production activities are successive approximate to the customization demands. Based on the above model assumptions, payment processes is divided into three main stages, deposit, payment in the interactive process and ultimate payment. Customization service process under customer participation is the embodiment of customers will, in which the exclusiveness of the material resources is growing. From the interactive between customers and manufacturers, customization degree and customer payment are significantly affected by situational factors, such as resource customization degree, customer perception etc., It is similar to ecological system composed by various populations in nature, which experiences development process from infancy to maturity. Therefore, we research the customization degree of customers to affect pay in stages to learn from Logistic curve in ecology theory, as shown in Figure 2.

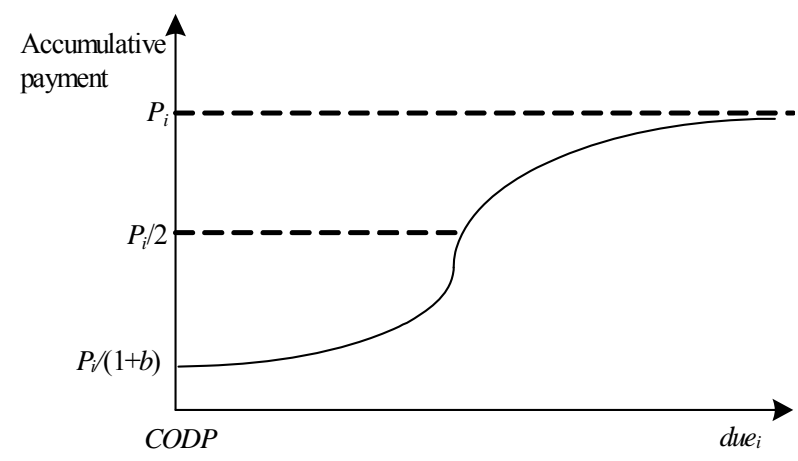

Figure 2. Logistic curve of customer payment in stages.
The logistic curve equation of the $j^{\text {th }}$ accumulative payment of the $i$ order at $t$ can be expressed as follows:

$$
p_{i, j, t}=P_{i}\left(1+b_{i} \exp \left(-a_{i} t\right)\right)^{-1}
$$

where $a_{i}$ and $b_{i}$ are unknown constants, $a_{i}>0$, $b_{i}>0$ and $b_{i} \neq 1$.

The logistic curve equation has the property as follows.

1) Substituting $t=0$ into (7), we can get customers the first payment amount at 0 , which is $p_{i, 1,0}=P_{i} /\left(1+b_{i}\right)$;

2) When the logistic curve reach the inflection point,

$$
\begin{aligned}
& \left.\quad p_{i, 2, t}^{\prime \prime}\right|_{t}=\frac{a_{i}^{2} b_{i} p_{i} \exp \left(-a_{i} t\right)}{\left(1+b_{i} \exp \left(-a_{i} t\right)\right)}\left(b_{i} \exp \left(-a_{i} t\right)-1\right)=0 \\
& \Rightarrow b_{i} \exp \left(-a_{i} t\right)=1 \\
& p_{i, 2, t}=P i / 2 \text { and } a_{i} t_{2}=\ln b_{i} .
\end{aligned}
$$

\subsection{Algorithms}

As the property of logistic curve for reference, we develop an algorithm for solving the parameters $a_{i}$ and $b_{i}$ in formula (7).

Step 1 by formula (6) to calculate CODP* $^{*}$, the initial payment for the $i$ order should be equal to $T C_{0 \rightarrow \mathrm{CDPP}^{*}}(1+r)^{t}$ according to the assumption (5). When $\mathrm{CODP}=\mathrm{CODP}^{*}$, customers place order (this moment $t=0$ ), Then the parameter $b_{i}$ is determined as following formula (8):

$$
\begin{aligned}
p_{i, 1, \mathrm{CODP}^{*}} & =P_{i} /\left.\left(1+b_{i} \exp \left(-a_{i} t\right)\right)\right|_{t=0}=P_{i} /\left(1+b_{i}\right) \\
& =\int_{0}^{\mathrm{CODP}^{*}}\left(p_{0}+c_{1}\right) d_{i}(1+r)^{t} \mathrm{~d} t .
\end{aligned}
$$

Step 2 the second payment is determined at $t_{2}$. When the Logistic curve reach inflection point, manufacturer has the largest operational risk, and thereafter gradually tends, so at this time manufacturer need withdrawal complete cost of the $i$ product, we can get formula (9) to determinate time $t_{2}$ :

$$
\begin{aligned}
p_{i, 2, t_{2}}=\frac{P_{i}}{2}= & {\left[\int_{\operatorname{CODP}^{*}}^{d u e_{i}} c_{2 i} d_{i}(1+r)^{-t} \mathrm{~d} t\right](1+r)^{t_{2}} } \\
& +p_{i, 1, \mathrm{CODP}^{*}}(1+r)^{t_{2}} .
\end{aligned}
$$

Step 3 the relationship between $t_{2}$ and $a_{i}$ is determined according to the property of the logistic curve at inflection point.

Step 4 the parameter $a_{i}$ is calculated and the Logistic curve equation of payment policy is determined.

Step 5 substituting $d u e_{i}$ into the Logistic curve equation of payment policy, we test the error, and require $b_{i} \exp \left(-a_{i}\right.$ due $\left._{i}\right)<0.01$.

The Logistic curve equation which parameters have 
been determined is accessible for manufacturer adopting postponement strategy to charge custom payment at any time.

\section{Empirical Analysis}

To illustrate the above models and algorithms, we investigate and analyze certain small and medium-sized electronic manufacturing enterprise in Shanghai, China, who customizes kinds of products according to certain rhythm. The production processes of adopting postponement strategy are given in Figure 3. Note that for the sake of confidentiality we shall keep the company and the product brand anonymous throughout the paper. The single type common electronic components are produced with three processes in the CODP upstream (Stage 1). The components have extensive applicability, which can be applied to mobile phone, LCD TV, radio. They are in buffer stock (CODP) waiting for customer orders arriving. According to target market specific requirements, those common electronic components can be customized to three different electronic products in three manufacturing centers, respectively.
In allusion to these customized products, if both manufacturer and customers consider the reasonable ROI is 20\%, other parameters are as shown in Table 1.

Because customers usually focus on customizing time from order placed to product completed, we introduce the parameter $d u e_{i}$ to express the time period. From the above models, we can get $L T_{i}=\mathrm{CODP}^{*}+d u e_{i}$.

\subsection{Results Analysis}

Substituting data above into formula (5), we can get $\mathrm{CODP}^{*}=2.62$ days .

According to the formula (8), the first payment of the order 1 is:

$$
\begin{aligned}
p_{1,1, \mathrm{CODP}^{*}} & =P_{1} /\left(1+b_{1}\right)=\int_{0}^{2.62}\left(p_{0}+c_{1}\right) d_{1}(1+r)^{t} \mathrm{~d} t, \\
& =15113.363
\end{aligned}
$$

then we can get $b_{1}=3$.

According to the formula (9), we can get $t_{2}=0.8$. Substituting $t_{2}$ into the property (2) of logistic curve equation, we can get $a_{1}=1.37$.

Then the logistic curve equation of customer payment

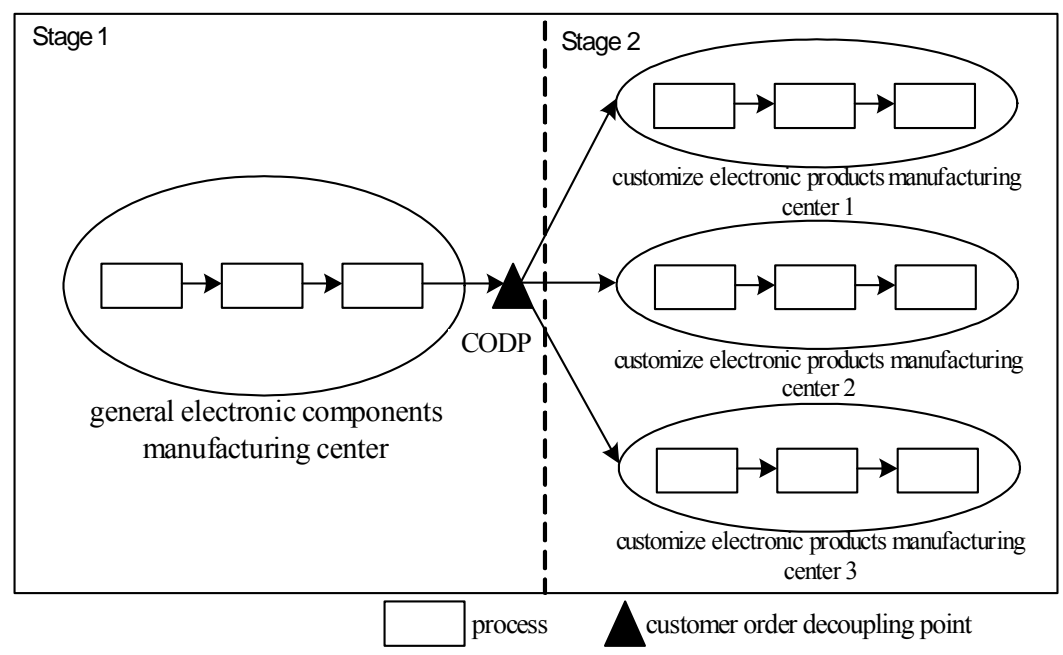

Figure 3. Production process of adopting postponement strategy.

Table 1. Production basic data of the electronic manufacturing enterprise a period of ten days.

\begin{tabular}{lcccc}
\hline \multirow{2}{*}{ basic data in the CODP upstream (Stage 1) } & \multicolumn{2}{c}{$\boldsymbol{p}_{\mathbf{0}}$ (\$/unit) } & \multicolumn{2}{c}{2} \\
\cline { 2 - 5 } & $\boldsymbol{c}_{\mathbf{1}}$ (\$/unit) & \multicolumn{2}{c}{1} \\
\cline { 2 - 5 } & $\boldsymbol{D}$ (unit) & \multicolumn{2}{c}{5000} \\
\hline & $\boldsymbol{i}$ & 1 & 2 & 3 \\
basic data in the CODP downstream (Stage 2) & $\boldsymbol{p}_{\boldsymbol{i}}$ (\$/unit) & 40 & 62 & 60 \\
& $\boldsymbol{d}_{\boldsymbol{i}}$ (unit) & 1500 & 1000 & 2500 \\
& $\boldsymbol{d u e}_{\boldsymbol{i}}$ (day) & 3 & 5 & 6 \\
\hline
\end{tabular}


for order 1 in stagy is

$$
p_{1, j, t}=P_{1}(1+3 \exp (-1.37 t))^{-1} \text {. }
$$

We test that $3 \exp \left(-1.37 d u e_{i}\right)=0.0031<0.01$ when $t=5$. The result shows that there is certain error, but acceptable.

Similarly, the calculation results of orders 2 and 3 are as shown in Table 2.

We can observe several conclusions from Table 2.

1) The first payment amount relies on the order quantities. Once the CODP ${ }^{*}$ is determined, $p_{i, 1, \mathrm{CODP}^{*}}$ depends on $d_{i}$ according to formula (8).

2) The second payment amount relies on the product unit price and quantity. From formula (9), we can get that $p_{i, 2 \text { CoDP* }}$ depends on $P_{i}$.

3) The parameters $a_{i}$ and $b_{i}$ have every relevance to dynamic cost of adopting postponement strategy.

\subsection{Sensitivity Analysis}

We assume the ROI is a constant in the above models and algorithms. However, market is volatile. When the ROI changes within a certain range, we simulate the changing rules of $\mathrm{CODP}^{*}$, and research how the CODP $^{*}$ affect the dynamic cost and payment policy. To investigate the impact of ROI on CODP* ${ }^{*}$, its values are systematically adjusted from $5 \%$ to $40 \%$ for the fixed value of 20\%. In Figure 4, we can think CODP* as a monotone decreasing concave function, as ROI increases. Namely, CODP ${ }^{*}$ is inclined to downstream of implementing postponement strategy along with the increase of ROI. The result is consistent with the reality. When some kind of product ROI is relatively low, manufacturers can choose to use make-to-order (MTO) fashion to improve products vitality in the market, while when ROI is high, manufacturers can choose to use make-to-stock (MTS) fashion to achieve economies.

According to formula (4), $T C_{0 \rightarrow \mathrm{CODP}^{*}}$ is associated with ROI and CODP*, we can calculate $T C_{0 \rightarrow \text { CODP* }^{*}}$ values when ROI change from $5 \%$ to $40 \%$. From Figure 4 , when ROI decreases, the upstream dynamic cost converted to COPD* increases. Because COPD* is ROI decreasing function, when $\mathrm{COPD}^{*}$ increases, the production

Table 2. The characteristic values of logistic curve equation of customer payment.

\begin{tabular}{cccc}
\hline & Order 1 & Order 2 & Order 3 \\
\hline$p_{i, 1, \text { CODP* }}$ & 15113.363 & 10075.575 & 25188.938 \\
$b_{i}$ & 3 & 5 & 5 \\
$t_{2}$ & 0.8 & 0.4 & 0.56 \\
$a_{i}$ & 1.37 & 4.02 & 2.87 \\
Logistic curve equation & $9000(1+3 \exp (-1.37 t))^{-1}$ & $10000(1+5 \exp (-4.02 t))^{-1}$ & $20000(1+5 \mathrm{exp}-2.87 t))^{-1}$ \\
error test & 0.0031 & $3.00555 \mathrm{E}-12$ & $5.34034 \mathrm{E}-10$
\end{tabular}

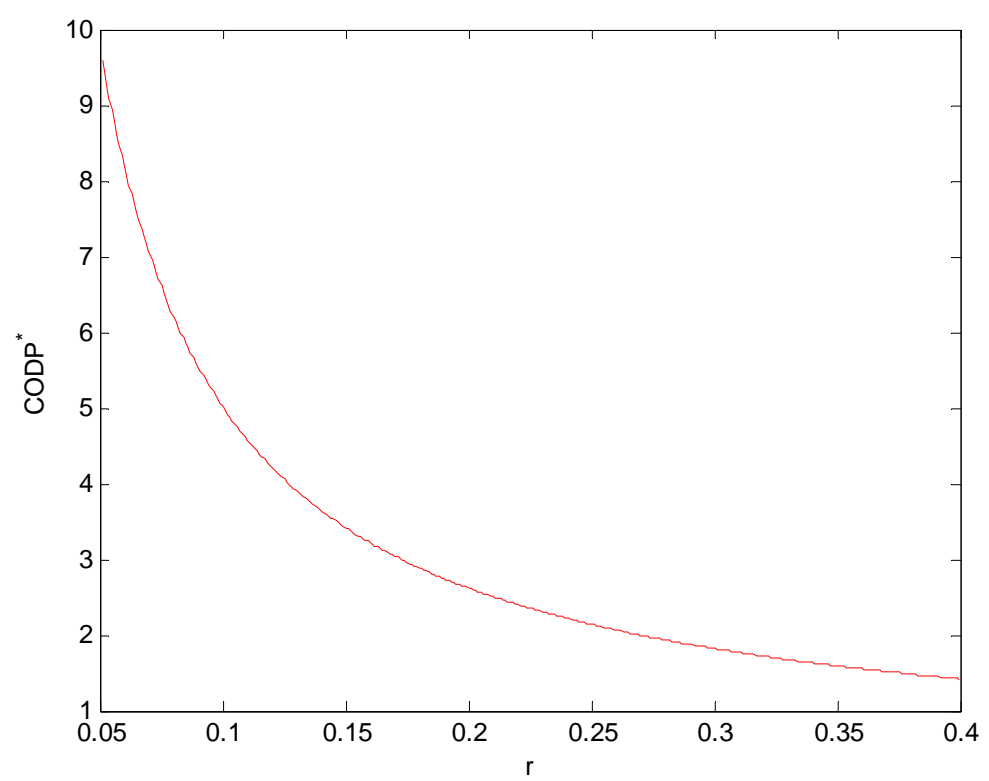

Figure 4. Relation between $r$ and CODP*. 
processes and the time are also increasing in the CODP upstream, it means that the dynamic cost of the CODP upstream is increasing.

From the Figure 5, we can show that $T C$ decreases with the increase of ROI. As the compensation to common component cost in the CODP upstream, $p_{1, i}$ also decreases with ROI increasing. Under the same ROI, $p_{1, i}$ is directly related with the order quantity, as shown in Figure 6.

According to formula (4), $T C_{\mathrm{CODP}^{*} \rightarrow d u e_{i}}$ is associated with ROI, due $e_{i}$ and CODP*. Under ${ }^{\mathrm{CODP}^{*}} \overrightarrow{d u e_{i}}$ and CODP* have been determined, we can calculate $T C_{\mathrm{CODP}^{*} \rightarrow d u e_{i}}$ values. From Figure 7, the CODP downstream dynamic cost converted to $\mathrm{CODP}^{*}$ is increasing with ROI increasing as shown in Figure 6.

\section{Concluding Remarks}

This paper uses the dynamic cost model and customer payment model to investigate three questions for manufacturers adopting postponement strategy. Unlike previous research that discusses the topic of potential or static

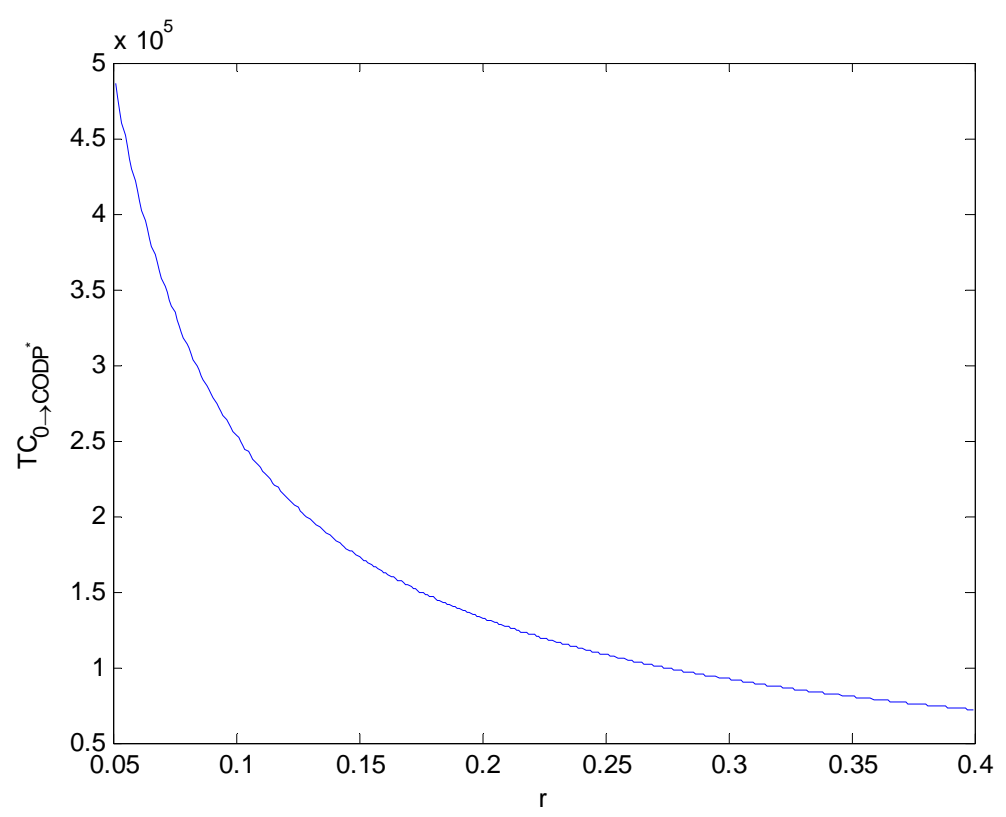

Figure 5. Relation between $r$ and $T C_{0 \rightarrow C O D P *}$

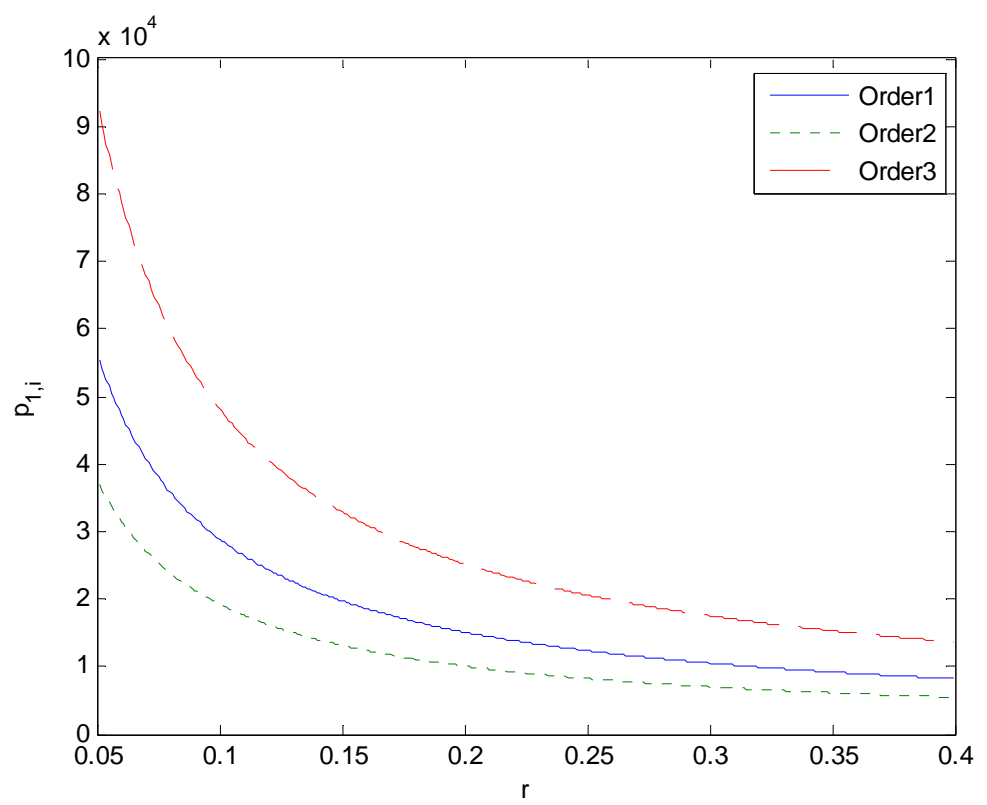

Figure 6. Relation between $r$ and $p_{1, i}$. 


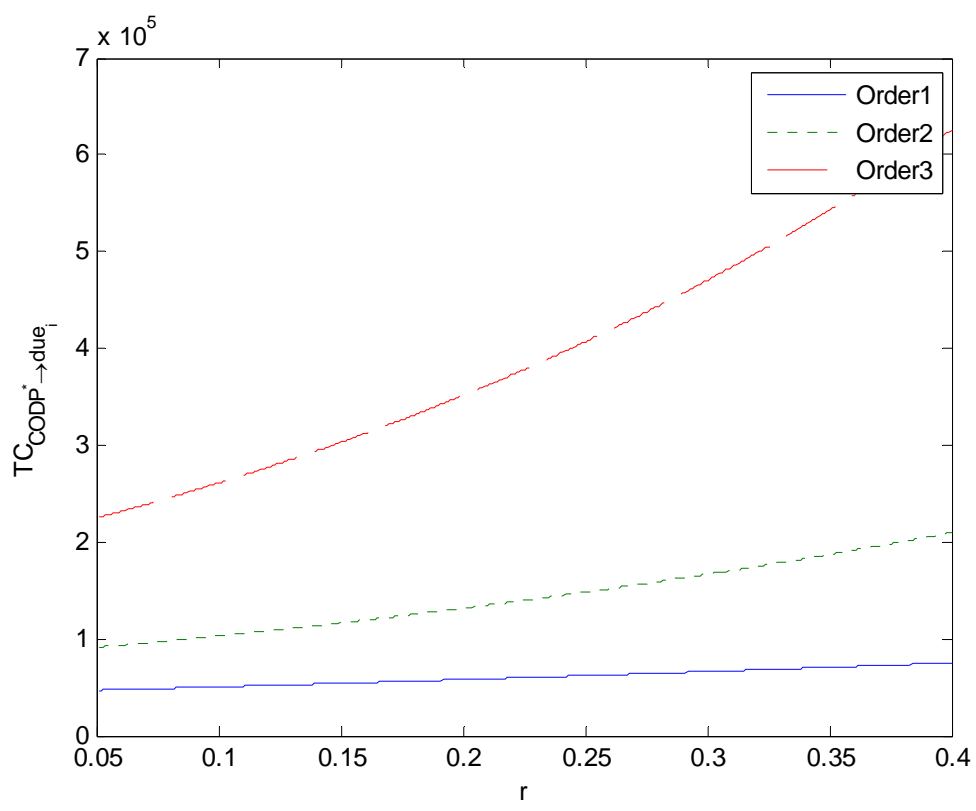

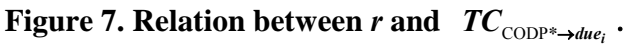

benefits or costs based on a single manufacturer perspective. We introduce the time value of money concept to construct the dynamic cost model considering the risk of material resource commonality diminishing in the CODP downstream. Then the COPD* positioning is researched under the coexistence of customized orders. Though model form is simple, tags are effective in revealing common component exclusiveness compensation. Then, the customer payment policy is given using the logistic curve equation. Based on the analysis, the algorithms to solve the models are developed. To validate the algorithms and models, an empirical analysis is presented. The research result shows that the algorithms and models are effective.

The limitations in this paper are as follows: 1) workin-process inventory costs both in the CODP upstream and downstream, and the buffer inventory costs at the CODP are neglected. However, postponement strategy is an important strategic means to coordinate contradiction between the absolute of market demand changes and relative stability of manufacturing system, there are costs. 2) The paper just considers the impact on the manufacturing system costs and risk compensation of the ROI, and assumes other parameters are constant such as the price of raw materials, processing time, etc. But they are changing in the actual production process. 3) Only single common component in the CODP upstream and three customized products in the CODP downstream are considered in empirical analysis. Moreover, multiple-component for customized products is universal in practice. Therefore, it would be interesting to study the above scenario in the future research.

\section{Acknowledgements}

This research was supported by the National Natural Science Foundation of China under grant No. 71172191, the Humanities and Social Sciences young Foundation of MOE of China under grant No. 10YJC630169.

\section{REFERENCES}

[1] J. Chod and N. Rudi, "Resource Flexibility with Responsive Pricing," Operations Research, Vol. 53, No. 3, 2005, pp. 532-548. doi:10.1287/opre.1040.0191

[2] A. O. Brown, H. L. Lee and R. Petrankan, "Xilinx Improves Its Semiconductor Supply Chain Using Product and Process Postponement," Interface, Vol. 30, No. 4, 2000, pp. 65-80. doi:10.1287/inte.30.4.65.11650

[3] R. I. Van Hoek, "The Rediscovery of Postponement a Literature Review and Directions for Research," Journal of Operations Management, Vol. 19, No. 2, 2001, pp. 161-184. doi:10.1016/S0272-6963(00)00057-7

[4] B. Yang, N. D. Burns and C. J. Backhouse, "Postponement: A Review and an Integrated Framework," International Journal of Operations \& Production Management, Vol. 24, No. 5, 2004, pp. 468-487. doi: $10.1108 / 01443570410532542$

[5] C. A. Boone, C. W. Craighead and J. B. Hanna, "Postponement: An Evolving Supply Chain Concept," International Journal of Physical Distribution \& Logistics Management, Vol. 37, No. 8, 2007, pp. 594-611. doi:10.1108/09600030710825676

[6] W. Zinn and D. J. Bowersox, "Planning Physical Distribution with the Principle of Postponement," Journal of Business Logistics, Vol. 9, No. 2, 1988, pp. 117-136. http://worldcat.org/oclc/4304746

[7] B. Yang, N. D. Burns and C. J. Backhouse, "The Appli- 
cation of Postponement in Industry," IEEE Transactions on Engineering Management, Vol. 52, No. 2, 2005, pp. 238-258. doi:10.1109/TEM.2005.845223

[8] J. Li, T. C. E. Cheng and S. Y. Wang, "Analysis of Postponement Strategy for Perishable Items by EOQ-Based Models," International Journal of Production Economics, Vol. 107, No. 1, 2007, pp. 31-38.

[9] S. W. Bibhushan, K. S. Bhoon and F. T. S. Chan, "Postponement Strategies for Re-Engineering of Automotive Manufacturing: Knowledge-Management Implications," International Journal of Advanced Manufacturing Technology, Vol. 39, No. 3-4, 2008, pp. 367-387. doi:10.1007/s00170-006-0679-Z

[10] D. Kisperska-Moron and A. Swierczek, "The Elected Determinants of Manufacturing Postponement within Supply Chain Context: An International Study," International Journal of Production Economics, Vol. 133, No. 1, 2011, pp. 192-200. doi:10.1016/j.ijpe.2010.09.018

[11] R. I. Van Hoek, "Postponed Manufacturing: A Case Study in the Food Supply," Supply Chain Management: An International Journal, Vol. 2, No. 2, 1997, pp. 63-75. doi:10.1108/13598549710166113

[12] H. L. Lee, C. Billington and B. Carter, "Hewlett-Packard Gains Control of Inventory and Service through Design for Localization," Interfaces, Vol. 23, No. 4, 1993, pp. 1-11. doi:10.1287/inte.23.4.1

[13] M. Reimann, "Accurate Response by Postponement," European Journal of Operational Research, Vol. 220, No. 3, 2012, pp. 619-628. doi:10.1016/j.ejor.2012.02.022

[14] E. M. Jewkes and A. S. Alf, "A Queueing Model of De- layed Product Differentiation," European Journal of Operational Research, Vol. 199, No. 3, 2009, pp. 734-743. doi:10.1016/j.ejor.2008.08.001

[15] H. L. Lee, "Effective Inventory and Service Management through Product and Process Redesign," Operations Research, Vol. 44, No. 1, 1996, pp. 151-159.

[16] H. L. Lee and C. Tang, "Modeling the Costs and Benefits of Delayed Product Differentiation," Management Science, Vol. 43, No. 1, 1997, pp. 40-53. doi: $10.1287 / \mathrm{mnsc} .43 .1 .40$

[17] C. Yeh and H.-C. Yang, "A Cost Model for Determining Dyeing Postponement in Garment Supply Chain," International Journal of Advanced Manufacturing Technology, Vol. 22, No. 1-2, 2003, pp. 134-140. doi:10.1007/s00170-002-1453-5

[18] H. Haapasalo, "Fast Production for Order Fulfillment: Implementing Mass Customization in Electronics Industry," International Journal of Production Economics, Vol. 90, No. 2, 2004, pp. 213-222. doi:10.1016/S0925-5273(03)00214-7

[19] J. Uskonen and A. Tenhiälä, "The Price of Responsiveness: Cost Analysis of Change Orders in Make-to-Order Manufacturing," International Journal of Production Economics, Vol. 135, No. 1, 2012, pp. 420-429. doi:10.1016/j.ijpe.2011.08.016

[20] J. C. P. Su, Y.-L. Chang and M. Ferguson, "Evaluation of Postponement Structures to Accommodate Mass Customization," Journal of Operations Management, Vol. 23, No. 3-4, 2005, pp. 305-318. doi:10.1016/j.jom.2004.10.016 